\title{
Mathematical Model for Calculating the Particle Size Distribution Fractal Dimension Based on Image Processing Method and Its Application
}

\author{
Lieshu LIN ${ }^{1 a}$, Chengbao WU ${ }^{1 b^{*}}$, Chuansheng LIU ${ }^{1 c}$, Zhenghua CHEN ${ }^{2 d}$ \\ ${ }^{1}$ School of Aircraft Maintenance Engineering, Guangzhou Civil Aviation College, Guangzhou \\ 510430, China \\ ${ }^{2}$ Maintenance Engineering Department, Guangzhou Baiyun International Airport Ground Servicing \\ LTD. Company, Guangzhou, 510470, China \\ aemail:linlieshu@caac.net, ${ }^{\mathrm{b} e m a i l}$ :wuchengbao@caac.net, ${ }^{\mathrm{c} e m a i l}$ :liuchuansheng@caac.net, ${ }^{\mathrm{d}} \mathrm{email}$ \\ :steffens@163.com
}

Keywords: Particle Size Distribution, Fractal Dimension, Image Processing Method, Mathematical Model

\begin{abstract}
A mathematical model for calculating the PSDFD based on image processing method was constructed and applied to calculate the PSDFD of hollow glass bead(HGB) filled in the polypropylene(PP) resin. The results shown that the PSDFD of the HGB in the PP resin was 1.370, and the correlation coefficientwas0.9665; the large correlation coefficient indicated that the HGB in the PP resin was fractal evidently; the mathematical model was correct and feasible.
\end{abstract}

\section{Introduction}

The inorganic powder with single size is mono-disperse. But, the powder of massive industrial product is composed of the particles with different size. That is, the particle size distribution (PSD) of a powder is not uniformly with a certain width. The wider the PSD is, the larger the poly-dispersity is, and the worse the uniform is. The main traditional methods for characterizing quantitatively the particle size distribution width (PSDW) of a powder included SPAN method [1], standard deviation method [2] [3] and diagram method [3] [4] [5]. The SPAN valve of SPAN method was used to characterize the PSDW; the larger the SPAN value is, the wider the PSD is, and otherwise, the narrower the PSD is. The SPAN method can be used to compare the PSDW of powders with same average size only [6], is not suitable for the powders with different average size. The standard deviation method is same with the SPAN method unsuitable for compare the PSDW of powders. The graphic method has two types: the differential type and integral-type. Both of them can be used to compare intuitively the PSD of two different powders. But, it can't characterize the PSDW quantitatively, so, it application scope is limited.

The methods mentioned above are based on the assumption that the PSD is continuous. As a matter of fact, the PSD of every powder is discrete and random. Tyler [7] and Yu [8] constructed a formula for calculating the PSDW of a powder based on the formula of basic definition of fractal, and used it to investigate the fractal characteristic of different powders and found that the PSD of soil particles, silica fine particles and cement was self-similar and fractal, the PSD fractal dimension (PSDFD) could characterize the PSDW quantitatively. In the last work, Wu [9] reckoned a mathematical model for calculating the PSDFD based on the Katz's work [10] and found that the PSDFD could be used to characterize the PSDW of cement quantitatively and the cement was fractal evidently. In this work, a new mathematical model for calculating the PSDFD based on image processing method will be constructed, and used to calculate PSDFD of powder.

\section{General Model for Calculating PSDFD}

The relationship between the particle size and number of the particles can be expressed as follows [11]: 


$$
F(x)=\frac{N(x)}{N_{0}}
$$

Where, $x$ is particle size of a certain fraction; $N(x)$ is the total number of the particles with the size less than $X ; N_{0}$ is the total number of the particles sample of the powder.

Because the fractal characteristic of the powder is self-similarity with limited layer, one can get[12] [13]:

$$
N(x)=N_{1}\left(\frac{x}{x_{\max }}\right)^{-D_{m}}
$$

Where $N_{1}$ is constant; $x_{\max }$ is the maximum particle size in the particles sample; $D_{m}$ is the PSDFD, $0 \leq D_{m} \leq 3$, the larger the $D_{m}$ is, the more complex the space structure of the particles is.

If $x=x_{\max }, N(x)=N_{0}, N_{1}=N_{0}$, to substitute $N_{1}=N_{0}$ into Eq. 2, one can get:

$$
N(x)=N_{0}\left(\frac{x}{x_{\max }}\right)^{-D_{m}}
$$

To substitute Eq.3 into Eq.1:

$$
F(x)=\left(\frac{x}{x_{\max }}\right)^{-D_{m}}
$$

The relationship between the particle size and mass distribution can be expressed as the following function: [14]:

$$
W(x)=\frac{M(x)}{M_{0}}
$$

Where $M(x)$ is total mass of the particles with particle size less than or equal to $x ; M_{0}$ is the total mass of the particles sample of the powder.

Totakederivative of Eq. 5and substitute the mass and volume function of the particle into it, one can get:

$$
d M(x)=\rho \cdot V(x) d N(x)
$$

Where $\rho$ is the density of the powder, $V(x)$ is the volume of the particles with particle size in the range of $(x, x+d x), d N(x)$ is the number of the particles with particle size in the range of $(x, x+d x)$.

To set the $k$ is volume shape factor of the smooth particle, one can get:

$$
V(x)=k \cdot x^{3}
$$

To substitute Eq.7 into Eq.6:

$$
d M(x)=\rho \cdot k \cdot x^{3} d N(x)
$$

To substitute Eq.8) into Eq.5 after taken derivative:

$$
d W(x)=\frac{\rho \cdot k \cdot x^{3} d N(x)}{M_{0}}
$$

Totakederivative of Eq. 1:

$$
d N(x)=N_{0} d F(x)
$$

To substitute Eq.10 into Eq.9:

$$
d W(x)=\frac{\rho \cdot k \cdot x^{3} N_{0} d F(x)}{M_{0}}
$$

To take derivative of Eq.4: 


$$
d F(x)=-D_{m} \cdot x_{\max }^{D_{m}} \cdot x^{-D_{m}-1} d x
$$

To substitute Eq.12 into Eq.11:

$$
d W(x)=\frac{-D_{m} \cdot \rho \cdot k \cdot x^{3} \cdot N_{0} \cdot x_{\max }^{D_{m}} \cdot x^{-D_{m}-1} d x}{M_{0}}
$$

To integrate the Eq.13:

$$
\int_{x_{\min }}^{x_{\max }} d W(x)=\int_{x_{\min }}^{x_{\max }} \frac{-D_{m} \cdot \rho \cdot k \cdot x^{3} \cdot N_{0} \cdot x_{\max }^{D_{m}} \cdot x^{-D_{m}-1}}{M_{0}} d x
$$

That is:

$$
W(x)=-\frac{k \cdot \rho \cdot N_{0} \cdot D_{m} \cdot x_{\max }^{D_{m}}}{M\left(3-D_{m}\right)} x^{3-D_{m}}+C
$$

To substitute the boundary condition equations, $W\left(x=x_{\max }\right)=1$ and $W\left(x=x_{\min }\right)=0$, into Eq.13:

$W(x)=\frac{x^{3-D_{m}}-x_{\min }^{3-D_{m}}}{x_{\max }^{3-D_{m}}-x_{\min }^{3-D_{m}}}$

As a general rule, $x_{\min }$ is a smaller number, and $x_{\max }$ is a large integer, $x_{\min } / x_{\max } \rightarrow 0$. Therefore, Eq. 16 can be simplified further:

$$
W(x)=\left(\frac{x}{x_{\max }}\right)^{3-D_{m}}
$$

To take the logarithm of Eq.17, and fit $\lg (W(x))$ and $\lg \left(\frac{x}{x_{\max }}\right)$, one can get a fitting equation:

$\mathrm{Y}=\mathrm{A}+\mathrm{B} \cdot \mathrm{X}$

Where $\mathrm{Y}$ is the $\lg (W(x)), \mathrm{X}$ is $\lg \left(\frac{x}{x_{\max }}\right), \mathrm{B}$ is the slope of the fitting equation, $3-D_{m}$ :

$$
D_{m}=3-B
$$

\section{Mathematical Model for Calculating PSDFD Based on the Image Processing Method}

It can be seen from Eq.17 that the general model is suitable for the case that the mass fraction of each size fraction of the particles sample of powder can be measured directly. But, once the inorganic powder was filled into the resin, the PSD and mass fraction of each size fraction of the powder would be changed because of the mixture, extrusion and injection of the plastic machinery and could not be measured directly. On certain conditions, one must calculate the PSDW or PSDFD on the basis of the image of the particles of the powder. How toresolvetheseproblems? It is necessary to build up a mathematical model for calculating PSDFD based on the image processing method.

Without lossofgenerality, the volume of a particle is in proportionto the product of the projection area and the biaxialarithmetic average size. The volume function of a particle can be expressed as follows [15]:

$$
v_{i}=k_{5} s_{i p} \frac{d_{i \max }+d_{i \min }}{2}
$$

Where $k_{5}$ is a constant in relation to the particle shape, $s_{i p}$ is the projection area of a single particle, $d_{i \max }$ is the maximum projection diameter of a single particle, $d_{i \min }$ is the minimum projection diameter. 
To set the mass of single particle as $w_{i}$, then:

$w_{i}=\rho_{i} v_{i}$

To substitute Eq. 20 into Eq.21:

$w_{i}=\rho_{i} k_{5} s_{i p} \frac{d_{i \max }+d_{i \min }}{2}$

To set the total mass of the $I^{\text {st }}$ fraction particles of the powder as $W_{\mathrm{I}}$ :

$W_{I}=\sum_{i=1}^{n} w_{i}$

If ignoring the difference of the density $\rho$ among the particles, and substitute Eq.22 into Eq.23:

$W_{I}=\sum_{i=1}^{n} \rho k_{5} s_{i p} \frac{d_{i \max }+d_{i \min }}{2}$

To set the total mass of the particlesfrom $1^{\text {st }}$ fraction to $I^{\text {st }}$ fraction as $W(x)$ :

$$
W(x)=\sum_{i=1}^{I} W_{I}
$$

To set the total mass of the particles sample of the powder as $W_{o}$ :

$W_{o}=\sum_{i=1}^{I_{\max }} W_{I}$

To substitute Eq.24 into Eq.25 and Eq.26, respectively:

$$
\begin{aligned}
& W(x)=\sum_{i=1}^{I} \sum_{i=1}^{n} \rho k_{5} s_{i p} \frac{d_{i \max }+d_{i \min }}{2} \\
& W_{o}=\sum_{i=1}^{I_{\max }} \sum_{i=1}^{n} \rho k_{5} s_{i p} \frac{d_{i \max }+d_{i \min }}{2}
\end{aligned}
$$

To substitute Eq.27 and Eq.28 into Eq.26:

$$
\sum_{i=1}^{I} \sum_{i=1}^{n} s_{i p} \frac{d_{i \max }+d_{i \min }}{2} / \sum_{i=1}^{I_{\max }} \sum_{i=1}^{n} s_{i p} \frac{d_{i \max }+d_{i \min }}{2}=\left(x / x_{\max }\right)^{3-D_{m}}
$$

The Eq.29 is the mathematical model for calculating PSDFD of powder, which takes the geometric features of the particle consideration.To take the logarithm of Eq.29, and Eq.30 is got:

$\lg \left(\sum_{i=1}^{I} \sum_{i=1}^{n} s_{i p} \frac{d_{i \max }+d_{i \min }}{2} \int_{i=1}^{I_{\max }} \sum_{i=1}^{n} s_{i p} \frac{d_{i \max }+d_{i \min }}{2}\right)=\left(3-D_{m}\right) \lg \left(x / x_{\max }\right) \quad\left(0<D_{m}<3\right)$

To set $\lg (W(x))=\lg \left(\sum_{i=1}^{I} \sum_{i=1}^{n} s_{i p} \frac{d_{i \max }+d_{i \min }}{2} / \sum_{i=1}^{I_{\max }} \sum_{i=1}^{n} s_{i p} \frac{d_{i \max }+d_{i \min }}{2}\right)$, fit $\lg (W(x))$ and $\lg \left(\frac{x}{x_{\max }}\right)$, one can get a fitting equation:

$$
Y=C+D X
$$

The slope of the fitting equation $D$ :

$$
\begin{aligned}
& D=3-D_{m} \\
& D_{m}=3-D
\end{aligned}
$$




\section{Application}

Figure 1 is the image of a kind of inorganic particle named hollow glass bead (HGB) filled into the polypropylene resin. It can be seen from the image that the HGB is spherical in shape and disperses in the matrix.

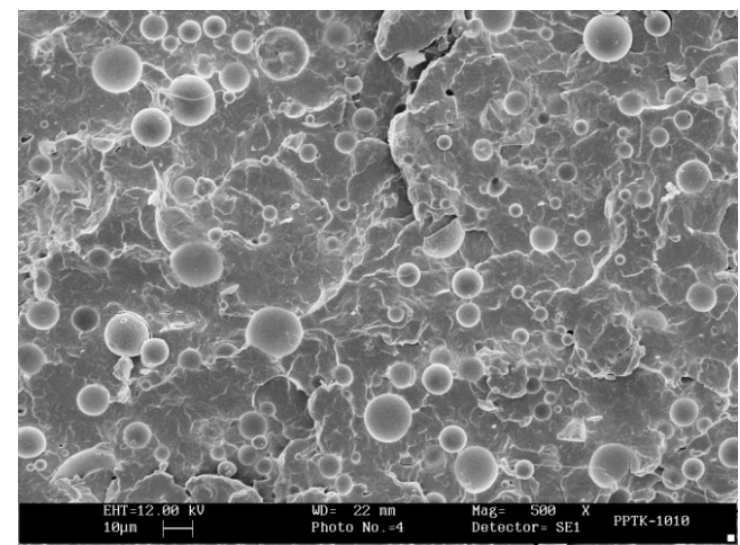

Fig.1 the imageof hollow glass bead filled in polypropylene composite

Before calculating $D_{\mathrm{m}}$ of HGB, the original SEM image shouldbe input to the computer firstly. Then, the originalphotographs were imported to image process software, ImageProcess-plus (IPP).the parameters of the particle, including $s_{i p}, d_{i \max }, d_{i \min }$, were measured by using the tools in the IPP. The differential distributionand cumulative distribution of hollow glass bead were shown in Figure 2.

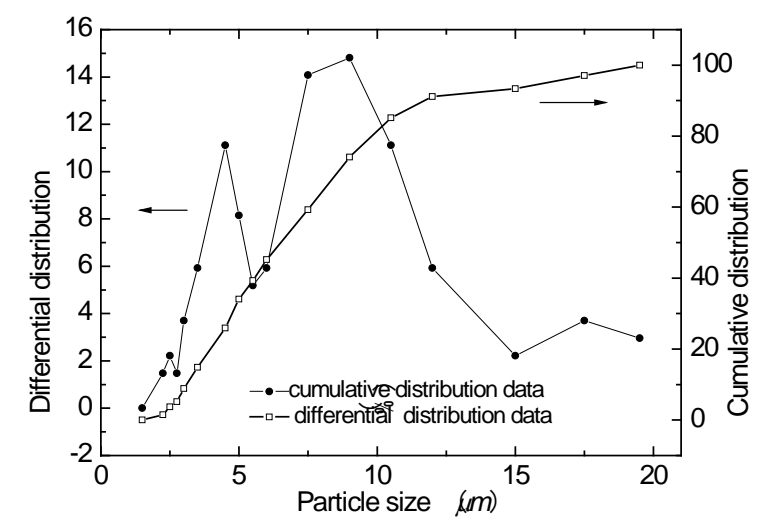

Fig.2particle size distribution of hollow glass bead in matrix

From Fig.2, we can see that the differential distribution of the HGB is binodal, and its cumulative distribution is $S$ type cure. In order to characterize the PSDW of the HGB quantitatively, the PSDFD of the HGB is calculated by using the Eq.29 and Eq.30 .The calculatingcurve of $D_{m}$ is expressed in Fig. 3.

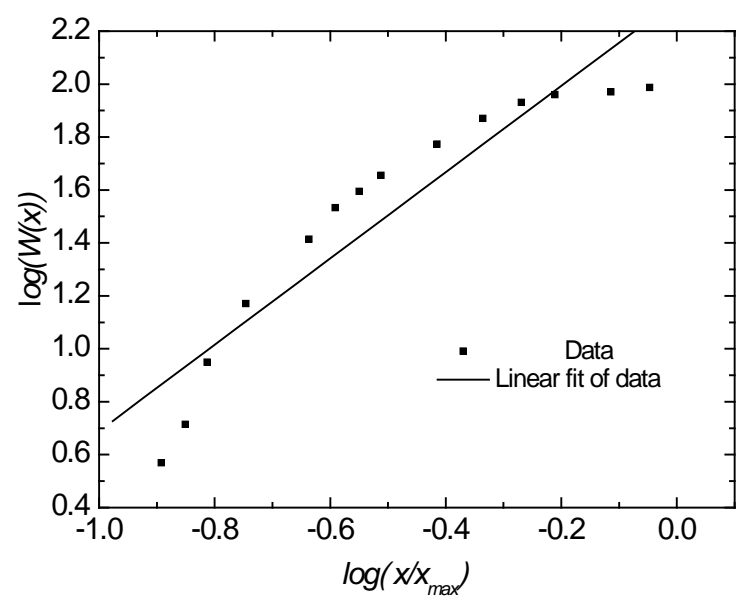

Fig.3 calculated curves for Dm of HGB 
It can be seen from the Fig.3 that the $\lg \left(\frac{x}{x_{\max }}\right) \sim \lg (W(x))$ curve is close to linear. Therefore, the data may be fitted bylinear regression analysis method and the regressionresult is following:

$y=2.3194+1.6305 x$

The correlation coefficient(R)is $0.9665, D_{m}$ is 1.370 . The correlation coefficient of the fitting linear line(Eq.34)is largerthan 0.97. In general, strong correlation coefficientindicates that the PSDW of HGB is fractal evidently. That is to say, the mathematical model for calculating PSDFD of powder based on the image processing method is correct and feasible.

\section{Conclusions}

PSDW is one of important parameters of powder; PSDFD can be used to characterize the PSDW quantitatively. A mathematical model for calculating the PSDFD based on image processing method was constructed. Its application indicated that the mathematical model was correct and feasible for calculating the PSDFD of powder.

\section{References}

[1] J.J. Torrajd, L.Illum, S.S. Daviss, Particle size and particle size distribution of albumin microspheres produced by heat and chemical stabilization, [J]. International Journal of Pharmaceutics, 1989, 51: 85-93.

[2] B. Turcsanyi, B. Pukanszky, F. Tudos, Composition dependence of tensile yield stress in filled polymers, [J]. Journal of Materials Science Letters, 1988, 7: 160-162.

[3]Handbook of Chemical Engineering Editorial Board. Handbook of Chemical Engineering(Vol.19)particle and particle system, [M]. 1989, 10.

[4] V.Khunova, J.Hurst, I.Janigova, et al. Plasma treatment of particulate polymer composites for analyses by scanning electron microscopy II. A study of highly filled polypropylene/calcium carbonate composites, [J]. Polymer Testing, 1999, 18: 501-509.

[5] X.D.Liu, D.C.Bao, W.M.Xue, et al, Preparation of uniform calcium alginate gel beads by membrane emulsification coupled with internal gelation, [J]. Journal of Applied Polymer Science, 2003, 87: 848-852.

[6] M.F.Han, C.B.Yang, Manifestation of Grain Characteristics of Nanocrystalline Powders, [J]. China Non-metallic Mining Industry Herald, 2003(4): 30-40.

[7] S.W.Tyler, S.W.Wheatcraft, Fractal scaling of soil particle-size distributions: analysis and limitations, [J]. Soil Science Society American Journal, 1992, 56: 326-369.

[8] K. Yu, Z.S. Zheng, Fractal Characteristics On Granularity Distribution Of Powder, [J]. Chinese Journal Of Materials Research,1995,18(3):539-542.

[9] C.B. Wu, B.T. Duan, Study on evaluation of particle size distribution of cement by fractal dimension, [J]. Cement, 2007(5): 8-11.

[10] A.J.Katz, A.H.Thompson, Fractal sandstone proes: implication for conductivity and pore formation, [J]. Physical Review Letters, 1985, 54: 1325-1328.

[11] S. Miwa, J. Hidaka, L. Yang, S.X. Xie. Handbook of Powder Engineering Test, [M].China Architecture \& Building Press, 1987.7.

[12] B.B.Mandelbort, The fractal geometry of nature, [M]. San Francisco: Freeman, 1982.

[13] J.Z. Zhang, Fractal, [M].Tsinghua University Press, 1995.9. 
[14] H.G. Lu, Introduction to powder technology, [M].Shanghai:TongJi University Press, 1998. 3.

[15] K.S. Wang, Fundamental Engineering Fluid and Powder Mechaic, [M]. China Metrology Publishing House, 2002. 9. 